\title{
Pulsations and orbital modulation of the intermediate polar 1WGA J1958.2+3232
}

\author{
A. J. Norton ${ }^{1}$, H. Quaintrell ${ }^{1}$, S. Katajainen ${ }^{2}$, H. J. Lehto ${ }^{2}$, K. Mukai ${ }^{3}$, and I. Negueruela ${ }^{4}$ \\ 1 Department of Physics and Astronomy, The Open University, Walton Hall, Milton Keynes MK7 6AA, UK \\ e-mail: A.J.Norton@open.ac.uk; H.Quaintrell@open.ac.uk \\ 2 Tuorla Observatory, Turku University, Väisäläntie 20, 21500 Piikkiö, Finland \\ e-mail: sekataja@astro.utu.fi; hlehto@astro.utu.fi \\ 3 NASA Goddard Space Flight Center, Laboratory for High Energy Astrophysics, Code 662, \\ Greenbelt, MD 20771, USA and Universities Space Research Association \\ e-mail: mukai@milkyway.gsfc.nasa.gov \\ 4 Observatoire Astronomique de Strasbourg, 11 rue de l'Université, 67000 Strasbourg, France \\ e-mail: ignacio@isaac.u-strasbg.fr
}

Received 20 November 2001 / Accepted 20 December 2001

\begin{abstract}
We present optical photometry, spectroscopy and photopolarimetry, as well as $A S C A$ X-ray observations, of the recently discovered intermediate polar 1WGA J1958.2+3232. Through the first detection of an optical beat frequency, we confirm the previously tentative suggestion that the spin period of the white dwarf is twice the $\mathrm{X}$-ray and optical pulsation period, which we also confirm in each case. We detect an orbital modulation in each of the $U, B, V, R$ and $I$ bands for the first time, and suggest that the true orbital period is the $-1 \mathrm{~d}$ alias of that previously suggested. We also confirm the presence of circular polarization in this system, detecting a variable polarization which has opposite signs in each of the $B$ and $R$ bands. The double peaked pulse profile and oppositely signed polarization pulses suggest that 1WGA J1958.2+3232 accretes onto both magnetic poles via a disc which is truncated relatively close to the white dwarf.
\end{abstract}

Key words. novae, cataclysmic variables - stars: magnetic fields - stars: individual - 1WGA J1958.2+3232

\section{Introduction}

Intermediate polars (IPs) are semi-detached interacting binaries in which a magnetic white dwarf accretes material from a Roche-lobe filling, usually late-type, main sequence companion star. The accretion flow from the secondary proceeds towards the white dwarf either through an accretion disc, an accretion stream, or some combination of both (known as disc overflow accretion), until it reaches the magnetospheric radius. Here the material attaches to the magnetic field lines and follows them towards the magnetic poles of the white dwarf. The infalling material that originates from an accretion disc takes the form of arc-shaped accretion curtains, standing above the white dwarf surface. At some distance from this surface, the accretion flow undergoes a strong shock, below which material settles onto the white dwarf, releasing X-ray to optical emission. Since the magnetic axis is offset from the spin axis of the

Send offprint requests to: A. J. Norton,

e-mail: A.J.Norton@open. ac.uk white dwarf, this gives rise to the defining characteristic of the class, namely X-ray (and usually optical) emission pulsed at the white dwarf spin period. Additionally, as the X-ray "beam" sweeps around the system, there is the possibility that some fraction of the emission will be reprocessed from structures such as the companion star or a bulge at the edge of an accretion disc. This will give rise to further optical emission pulsed at the lower orbital sideband of the spin frequency, namely the spin frequency of the white dwarf in the reference frame of the binary. Several IPs show a dominant optical pulsation at this orbital sideband frequency. X-ray pulsation at the orbital sideband frequency is due to an intrinsic modulation arising from pole-switching in the case of stream-fed accretion. Several IPs show this type of modulation (at least at some epochs) too. About twenty confirmed intermediate polars are now recognised with a similar number of candidate systems having been proposed. Comprehensive reviews of various aspects of their behaviour are given by Patterson (1994), Warner (1995), Hellier (1995; 1996) and Norton (1995). 


\section{Observational history}

1WGA J1958.2+3232 was first identified as a $\sim 12 \mathrm{~min}$ X-ray pulsator by Israel et al. (1998) from archival ROSAT PSPC light curves. Israel et al. (1999) then identified the optical counterpart to the system as a 16th magnitude star, and claimed it was of spectral type B0Ve, indicating that the system was a Be/X-ray binary. Negueruela et al. (2000) subsequently showed that this optical counterpart had a spectrum consistent with that of a magnetic cataclysmic variable - an intermediate polar. Uslenghi et al. (2000) later obtained time resolved white-light optical photometry of the star and discovered a $\sim 12$ min optical modulation with the same period as the X-ray modulation. They also saw some evidence that the pulse period may be twice this value. Next, Zharikov et al. (2001) obtained further time resolved $R$-band photometry and spectroscopy from which they detected a possible orbital period of $4.36 \mathrm{~h}$ as well as confirming the pulsation period as $733 \mathrm{~s}$. Most recently, Uslenghi et al. (2000) detected circular polarization from the source in the $R$ and $I$ bands, with evidence for possible modulation of the polarization at twice the previously observed pulsation period.

\section{Observations and data reduction}

\subsection{Optical photometry}

Time resolved photometric data were obtained at the $1 \mathrm{~m}$ Jacobus Kapteyn Telescope (JKT) between 9-15 July 2000, using the SITe2 CCD and UBVRI filters. For the majority of the observations, the CCD was windowed to readout a $900 \times 400$ pixel region around the target, corresponding to a field-of-view of $4.5^{\prime} \times 2.0^{\prime}$. Exposures in different filters were interleaved each night, with a dead-time between exposures (for readout and filter change) of 3040 s. Landolt standard fields were also observed through the relevant filters on each night. The observing log is shown in Table 1.

Bias subtraction and flat-fielding were carried out using routines in IRAF on all target and standard frames. Aperture photometry using further IRAF routines was then carried out on the target as well as a number of near-by comparison stars, indicated in Fig. 1. Lightcurves containing differential photometry of 1WGA J1958.2+3232 with respect to each of the comparison stars were constructed. Extinction coefficients were then derived for each filter used on each night by examining the variation in observed magnitude of the comparison stars as a function of airmass. By combining these with the observations of Landolt standards, this in turn allowed the derivation of zero points in each filter for each night. Finally, these corrections were applied to the lightcurves of the target, resulting in the calibrated lightcurves shown in Fig. 2.
Table 1. Observation log for $J K T$ photometry.

\begin{tabular}{|c|c|c|c|}
\hline Night & Filters & Exposures/s & No. of frames \\
\hline 9 July & $U$ & 600 & 5 \\
\hline \multirow[t]{4}{*}{2000} & $B$ & 60 & 7 \\
\hline & $V$ & 60 & 6 \\
\hline & $R$ & 60 & 7 \\
\hline & $I$ & 60 & 7 \\
\hline 10 July & $B$ & 60 & 64 \\
\hline \multirow[t]{2}{*}{2000} & $V$ & 60 & 10 \\
\hline & $V$ & 30 & 55 \\
\hline 11 July & $V$ & 30 & 104 \\
\hline 2000 & $R$ & 30 & 101 \\
\hline 12 July & $V$ & 30 & 98 \\
\hline 2000 & $I$ & 30 & 97 \\
\hline 13 July & $B$ & 60 & 85 \\
\hline 2000 & $V$ & 30 & 85 \\
\hline 14 July & $V$ & 30 & 126 \\
\hline 2000 & $R$ & 30 & 126 \\
\hline 15 July & $U$ & 600 & 4 \\
\hline \multirow[t]{2}{*}{2000} & $U$ & 300 & 15 \\
\hline & $V$ & 30 & 27 \\
\hline
\end{tabular}

\subsection{Optical photo-polarimetry}

Time resolved $B$ - and $R$-band circular photopolarimetry of 1WGA J1958.2+3232 was obtained at the $2.56 \mathrm{~m}$ Nordic Optical Telescope (NOT) between the nights of 28 February and 2 March, 2001. The Andalucia Faint Object Spectrograph and Camera (ALFOSC) was used with a 1/4-waveplate and a calcite block. The o and e images of the star were separated by 15 arcsec such that the images of 1WGA J1958.2+3232 do not overlap with other stars in the field.

The $R$-band data of 1 WGA J1958.2+3232 were obtained on 1 March 2001 between UT 5.94 and UT 6.81, covering 2.14 spin cycles whilst the $B$-band data were obtained on 3 March 2001 between UT 5.86 and UT 6.78, covering 2.25 spin cycles. The seeing was between 1 and 1.5 arcsec on both nights and exposure times were $58 \mathrm{~s}$ for the $B$-band and $45 \mathrm{~s}$ for the $R$-band images. On each night all the images were taken as one long sequence of exposures, giving a time-resolution of less than one minute.

The CCD-frames were bias-subtracted and flat-fielded with standard IRAF-routines and aperture photometry was carried out with DAOPHOT (Stetson 1992). Measurements were also made for some other bright stars in the field of 1WGA J1958.2+3232 to calibrate the zero-point of 


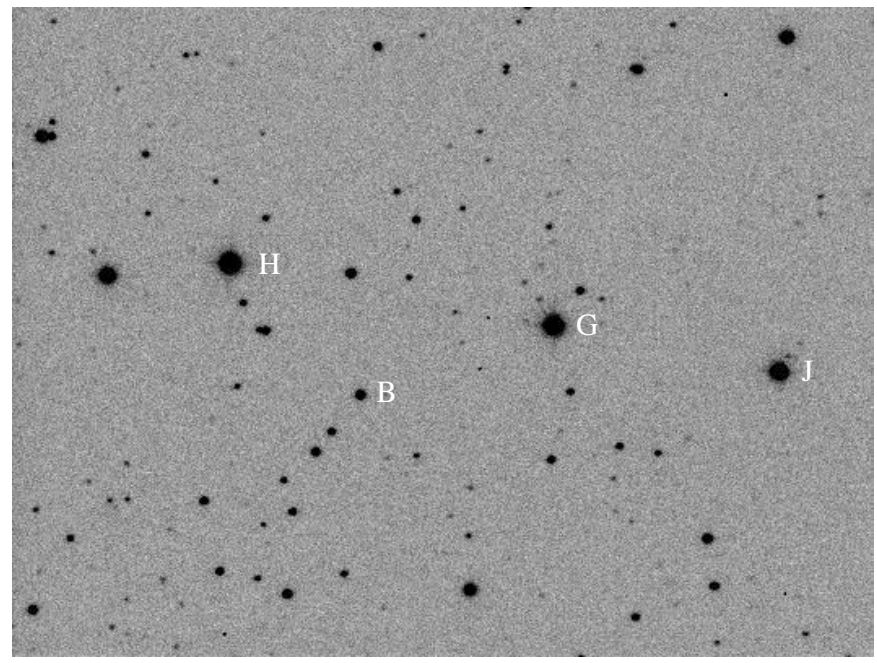

Fig. 1. A $V$-band image of the 1WGA J1958.2+3232 field covering $\sim 2.6^{\prime} \times 2.0^{\prime}$ obtained using the JKT. Following the nomenclature in Israel et al. (1999) and Uslenghi et al. (2000), the target is star $B$ whilst stars $H$ and $J$ were used for differential photometry; star $G$ may be variable.

the circular polarization. The sign of the circular polarization was checked by observing polarization standards Grw $+70^{\circ} 8247$ and LP 790-29. The instrumental polarization was found to be slightly dependent on the position of the star in the CCD-image. Corrections $(1.2 \%$ in the $B$-band, and $0.9 \%$ in the $R$-band) for instrumental polarization were made based on measurements of unpolarized field stars near the position of the object in the CCD-frame.

\subsection{Optical spectroscopy}

Two spectra of 1WGA J1958.2+3232, each spanning the wavelength range $\sim 3600-6700 \AA$, were obtained at the $2.5 \mathrm{~m}$ Isaac Newton Telescope (INT) on 21 July 2000 using the Intermediate Dispersion Spectrograph, $500 \mathrm{~mm}$ camera, R150V grating and TEK5 CCD. This combination yields a dispersion of $3.17 \AA$ pixel $^{-1}$. The exposure time for each spectrum was $1800 \mathrm{~s}$ and the two exposures began at 02:02 UT and 02:34 UT respectively. The resulting frames were bias subtracted then flat-fielded using tungsten lamp flats; the spectra were optimally extracted and calibrated using $\mathrm{CuAr}$ arcs. Figure 3 shows the average spectrum obtained, with the continuum normalised to unity, and emission lines identified.

The average INT spectrum shows a similar range of emission lines to that reported by Negueruela et al. (2000). The Balmer series lines up to at least $\mathrm{H} \zeta$ are all in emission, as are lines due to HeI ( $4472 \AA, 4922 \AA, 5876 \AA)$ and HeII (4686 $\AA, 5412 \AA)$. Unlike the spectra reported by Negueruela et al. (2000) however, the lines are not double peaked.
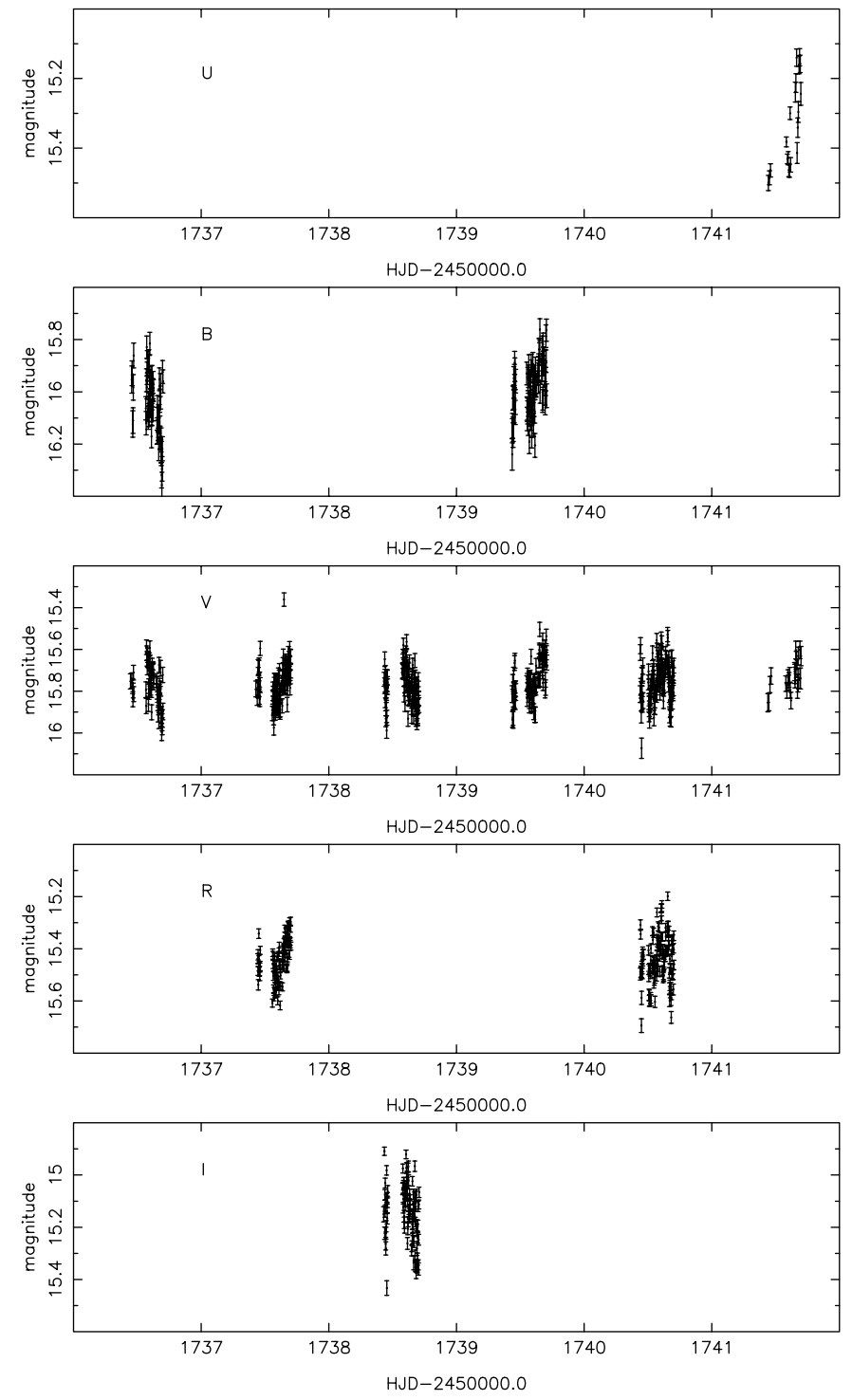

Fig. 2. The JKT $U, B, V, R$ and $I$-band lightcurves of 1WGA J1958.2+3232 for last six nights of the observing run.

\subsection{X-ray observations}

We have extracted the $A S C A$ (Tanaka et al. 1994) data of 1WGA J1958.2+3232 from the HEASARC. The observation was carried out on 1998 May 15 over an elapsed time of about 15.5 hrs. Data were taken with all 4 co-aligned instruments, two GIS's and two SIS's, all in standard configuration. We have applied the standard screening criteria (excluding Earth occultation, passages through the South Atlantic Anomaly, and times of high background), resulting in $\sim 30 \mathrm{ksec}$ of good data in each instrument. We have extracted light curves in $16 \mathrm{~s}$ bins from each instrument in low (0.4-2 keV for SIS, 0.7-2 keV for GIS) and high $(2-10 \mathrm{keV})$ energy bands, both from the source and from nearby source-free background region. We have scaled the latter using the detector areas, and subtracted from the former, to create net light curves; we have then summed the net light curves in the low and the high bands, and also created a total $(0.4 / 0.7-10 \mathrm{keV})$ light curve. 


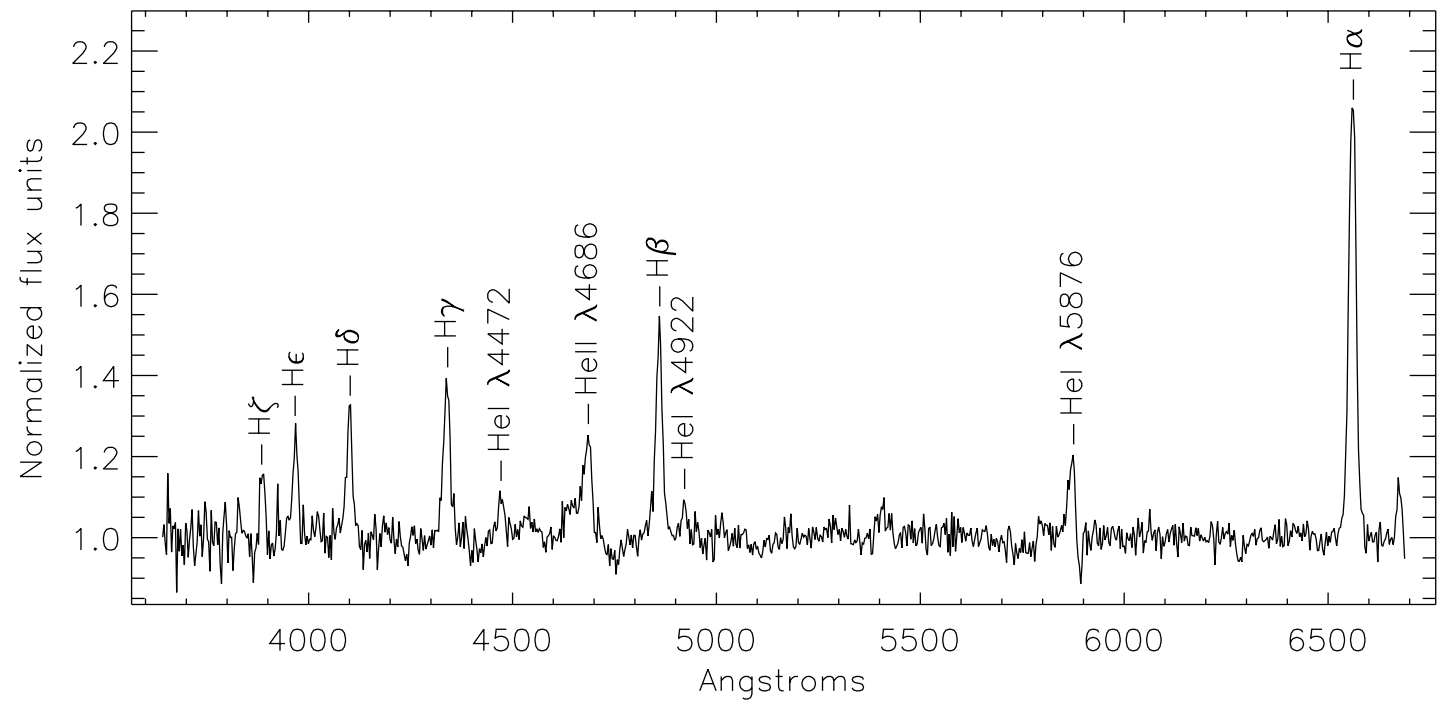

Fig. 3. The average INT spectrum of 1WGA J1958.2+3232 with emission lines identified.

Table 2. Average magnitudes and colours of possibility that one of the short periods is related to a 1WGA J1958.2+3232 from JKT data.

\begin{tabular}{lr}
\hline Filter & Magnitude \\
\hline$U-V$ & $-0.4 \pm 0.1$ \\
$B-V$ & $0.25 \pm 0.08$ \\
$V$ & $15.76 \pm 0.09$ \\
$V-R$ & $0.31 \pm 0.07$ \\
$V-I$ & $0.6 \pm 0.1$ \\
\hline
\end{tabular}

\section{Results}

The average magnitudes and colours of 1WGA J1958.2+3232 derived from our seven nights of JKT data are listed in Table 2. Formal uncertainties are dominated by the intrinsic variability of the source. We note that these magnitudes are consistent with the $U B V$ values reported by Uslenghi et al. (2000) and obtained about 1 year before our data.

\subsection{Optical power spectra}

The JKT lightcurves in magnitudes were converted into flux units prior to time series analysis. Figure 4 shows the power spectrum of the longest dataset, i.e. the $V$-band lightcurve, in two panels containing the low and high frequency regimes. Frequencies corresponding to the peaks were measured by fitting Gaussian profiles to the relevant portions of the power spectra.

The power spectrum is dominated by three sets of signals: one set centred around a frequency of $\sim 117.8 \mathrm{~d}^{-1}$; one set around $\sim 55.5 \mathrm{~d}^{-1}$; and a final set around $\sim 5.5 \mathrm{~d}^{-1}$. Each set of peaks consists of a series of sidebands due to daily aliases either side of a central strong peak. Clearly these three sets of frequencies are related to the pulse period, roughly twice that period, and the orbital period of the system as previously detected. There is also the beat between the white dwarf spin and system orbital periods. However, it is important to note that the strongest peaks in each of the three sets are not harmonically related to each other. A solution to the identification of the frequencies is as follows.

- We identify the strongest peak in the highest set of frequencies with twice the spin frequency of the white dwarf, i.e. $2 \omega=117.819 \pm 0.005 \mathrm{~d}^{-1}$ or $P_{\text {spin }} / 2=$ $733.33 \pm 0.03 \mathrm{~s}$. This is identical with the pulse period previously detected by Israel et al. (1998) and Uslenghi et al. (2000);

- We identify one of the peaks in the middle set of frequencies as the beat frequency between the white dwarf spin and system orbital frequencies. However, exactly which of the peaks is to be identified with the beat frequency is not immediately clear;

- We identify one of the peaks in the lowest set of frequencies with the orbital frequency of the system. However, as above, exactly which of the peaks is to be identified with the orbital frequency is not immediately clear.

The strongest peak in lowest set of frequencies corresponds roughly with the orbital period claimed by Zharikov et al. (2001), i.e. $\Omega \sim 5.5 \mathrm{~d}^{-1}$ or $P_{\text {orb }} \sim 4.4$ h. However, if this is chosen, it implies that the beat frequency is the -2 day alias of the strongest peak in the middle set of frequencies, and this peak clearly has a very low significance since there are many other peaks of comparable strength in the spectrum. Conversely, if the strongest peak of the middle set is the beat frequency, it implies that the orbital frequency is the -2 day alias of the strongest peak in the lowest set. This peak too has a relatively low significance. Given the relative weakness of the -2 day aliases of the strongest peaks in each of these two sets of frequencies, the most likely combination of peaks to represent the system frequencies is as follows. 


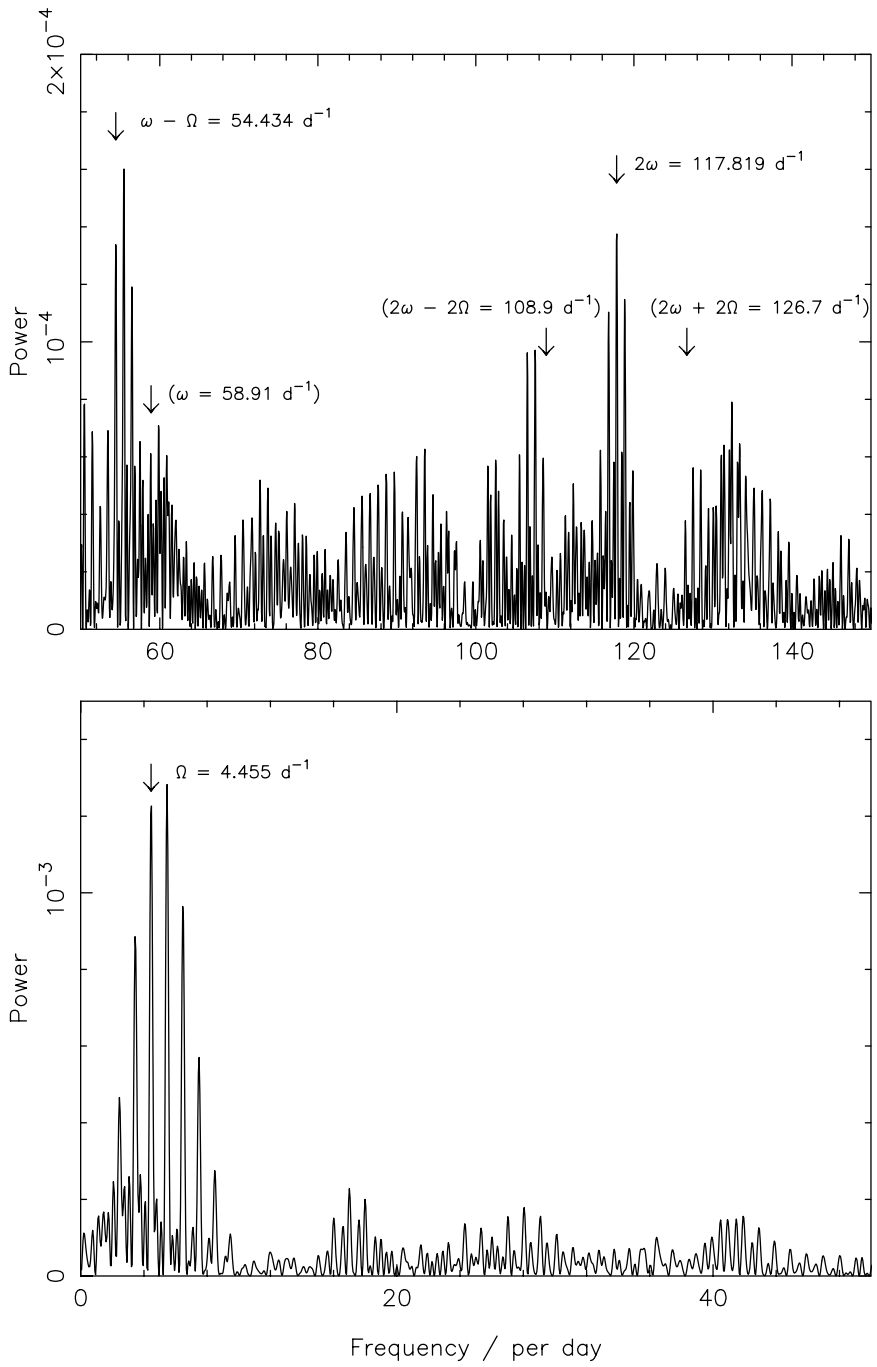

Fig. 4. The power spectrum of the JKT $V$ band lightcurve of 1WGA J1958.2+3232. The upper panel shows the frequency range $50-150 \mathrm{~d}^{-1}$, whilst the lower panel shows the frequency range $0-50 \mathrm{~d}^{-1}$. Note the change in vertical scale by a factor of 7.5 between the two panels. The proposed spin, orbital and beat frequencies are indicated.

- The -1 day alias of the strongest peak in the lowest set of frequencies is the orbital frequency of the white dwarf, $\Omega=4.455 \pm 0.005 \mathrm{~d}^{-1}$ or $P_{\text {orb }}=5.387 \pm 0.006 \mathrm{~h}$. This is the -1 day alias of the orbital period previously detected by Zharikov et al. (2001);

- The -1 day alias of the strongest peak in the middle set of frequencies is the beat frequency between the white dwarf spin and the orbit $(\omega-\Omega)=54.434 \pm$ $0.005 \mathrm{~d}^{-1}$ or $P_{\text {beat }}=1587.24 \pm 0.14 \mathrm{~s}$.

More extreme aliases combinations are probably unlikely, since the power at these aliases is low, although such combinations are possible in principle.

Figure 5 shows the $J K T$ power spectra for the low frequency regime in each of the $U, B, V, R$ and $I$ bands. The orbital frequency detected in the $V$-band (indicated by the dotted line) is also seen clearly in the $R$ and $B$-band power spectra. Broad peaks in the $I$ and $U$-band power spectra
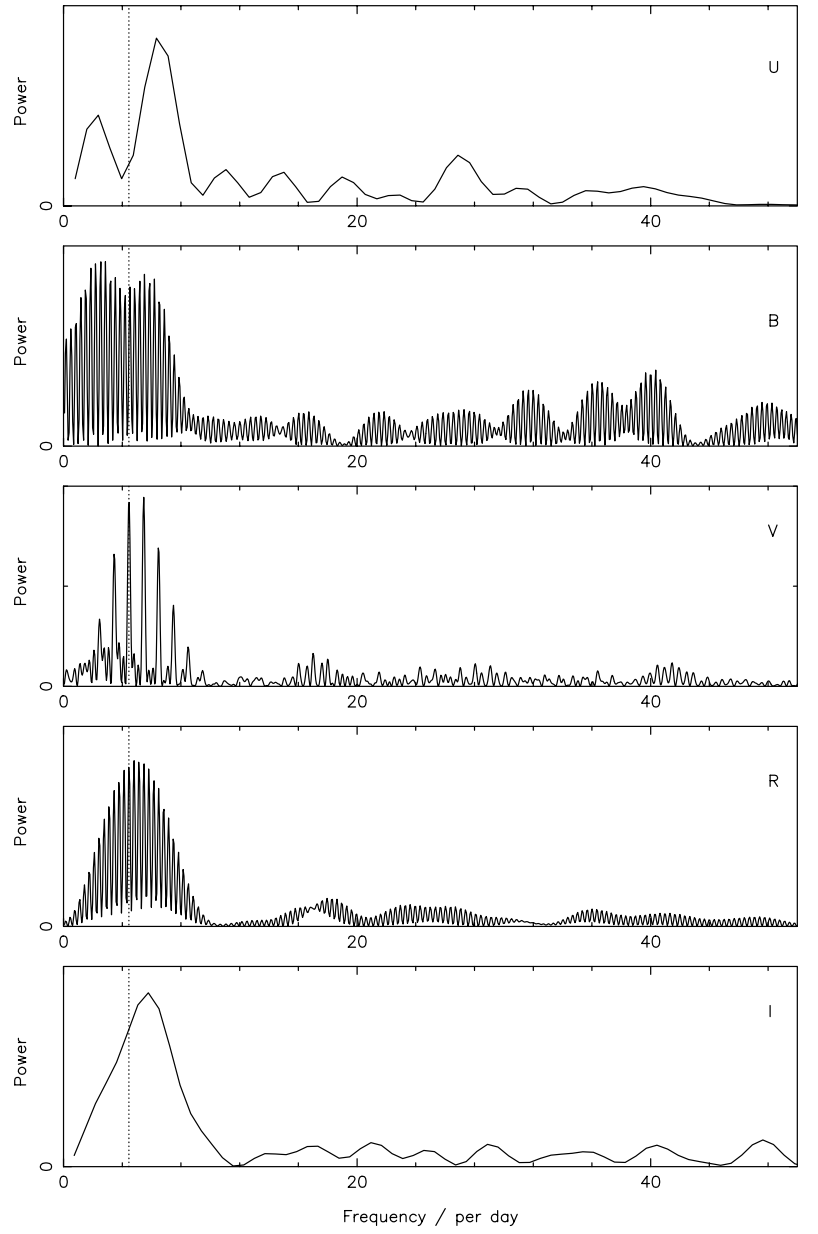

Fig. 5. The low frequency power spectra of the JKT $U, B, V$, $R$ and $I$ band lightcurves of 1WGA J1958.2+3232. The dotted line indicates the proposed orbital frequency $(\Omega)$. Vertical axis scales are arbitrary.

are consistent with the same period, possibly shifted to higher frequencies, but this apparent shift is probably an artifact of the small amount of data in these bands. We therefore detect the orbital period of the system in all five wave-bands.

Figure 6 shows the same $J K T$ power spectra, but this time in the higher frequency regime, for all five wavebands. Both the $B$ and $I$-band lightcurves contain signals consistent with twice the spin frequency (i.e. $2 \omega$ ) seen in the $V$ band, indicated by the right-most dotted line. In the $U$ band, broad peaks appear either side of this location, whilst in the $R$ band, no signal is apparent. The two leftmost dotted lines indicate the proposed spin frequency $(\omega)$ and the beat frequency $(\omega-\Omega)$. The beat frequency is not clearly detected in any waveband other than $V$, whilst the spin frequency itself may be detected in the $R$ band, and possibly in the $B$ band. 

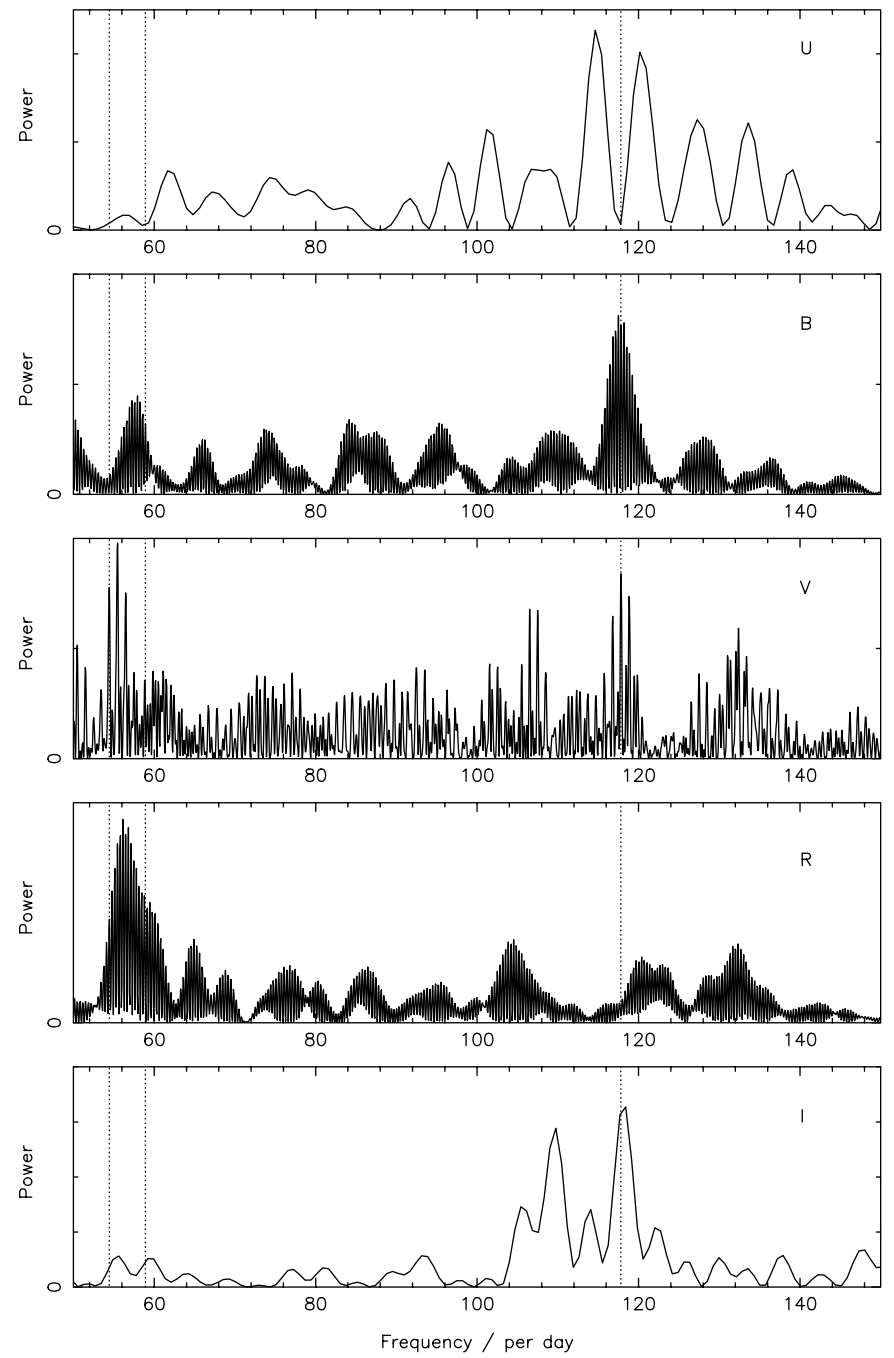

Fig. 6. The high frequency power spectra of the $J K T U, B, V$, $R$ and $I$ band lightcurves of 1WGA J1958.2+3232. Working from the right, the dotted lines indicate twice the proposed spin frequency $(2 \omega)$, the proposed spin frequency $(\omega)$, and its orbital sideband $(\omega-\Omega)$. Vertical axis scales are arbitrary.

\subsection{Ephemerides}

From the phasing of the peaks in the Fourier transform of the $J K T V$-band lightcurve, we are able to determine spin and orbital ephemerides, based on our observations of:

Half the spin period:

$\mathrm{HJD}=2451739.318445(2)+0.0084876(4) E$.

Orbital period:

$$
\mathrm{HJD}=2451739.36435(2)+0.2245(3) E
$$

where the zero phase is at minimum light in each case.

We can also combine our determination of the pulse period with earlier measurements to estimate the spin down rate of the white dwarf. The most accurate previous determination of the pulse period is that by Uslenghi et al. (2000) who measured it as $733.24 \pm 0.02 \mathrm{~s}$ in June 1999.
Combined with our determination of $733.33 \pm 0.03 \mathrm{~s}$ from July 2000, this yields a rate of change for the period of:

$\dot{P}=(2.6 \pm 1.2) \times 10^{-9}$.

Of the other confirmed intermediate polars, PQ Gem, V1223 Sgr and AE Aqr are also spinning down, but their $\dot{P}$ values are at least two orders of magnitude smaller than this (e.g. Patterson 1994; see also http://lheawww.gsfc.nasa.gov/users/mukai/iphome/ iphome.html). Similarly, the systems which are spinning up (BG CMi, DQ Her, EX Hya, AO Psc, GK Per) have $\dot{P}$ values which are also of the order $10^{-11}$. FO Aqr has been seen to change from spin down to spin up over the last several years, but again the rate of period change is much smaller than the apparent change in 1WGA J1958.2+3232. Indeed the inferred $\dot{P} / P$ for 1 WGA $\mathrm{J} 1958.2+3232$ is of the order of $10^{-4} \mathrm{yr}^{-1}$, which is what is expected for a neutron star accretor rather than a white dwarf (e.g. King \& Williams 1983). It is likely therefore that the measured pulse periods for 1WGA J1958.2+3232 are not as accurate as claimed.

\subsection{Optical modulation profiles}

The $J K T U, B, V, R$ and $I$ lightcurves were folded, using the ephemerides above, at half the proposed spin period, at the proposed beat period, and at the proposed orbital period of the system. After folding and binning, fluxes were converted back to magnitudes, yielding the profiles shown in Fig. 7. The orbital modulation is clearest in the $V$-band, but a similar profile with similar phasing is also apparent in the $B$ and $R$-bands. The orbital profiles in the $U$ and $I$-bands are more sparse but also consistent with the $V$-band profile. The modulation at half the proposed white dwarf spin period is clearest in the $B$ and $V$-bands. The other bands are too noisy to draw any conclusions. Although an apparent modulation is seen in the $I$-band, it is out of phase with those in the $B$ and $V$-bands. Of the folds at the beat period, only those in the $B$ and $V$ bands are convincing, although the profile in the $R$ band is also reasonable. Much of the noise evident in all the $U$ and $I$ band folded profiles is due to the relatively small amount of data in these bands.

\subsection{Optical polarimetry}

The extent of the polarimetric lightcurves are not sufficient to allow any period searching. Instead, the reduced $B$ - and $R$-band circular polarization data and light curves were averaged into 12 phase bins over one spin cycle $\left(P_{\text {spin }}=1466 \mathrm{~s}\right)$, according to the ephemeris in Sect. 4.2. The light curves shown in Figs. 8a and 9a represent differential magnitudes with respect to the bright field star $\mathrm{H}$ (see Fig. 1). We note that these $B$ and $R$ band pulse profiles appear to show a single peak when folded on the inferred spin period, rather than the double peaked pulse profile that would be expected from the photometry reported previously and elsewhere in this paper. However, 

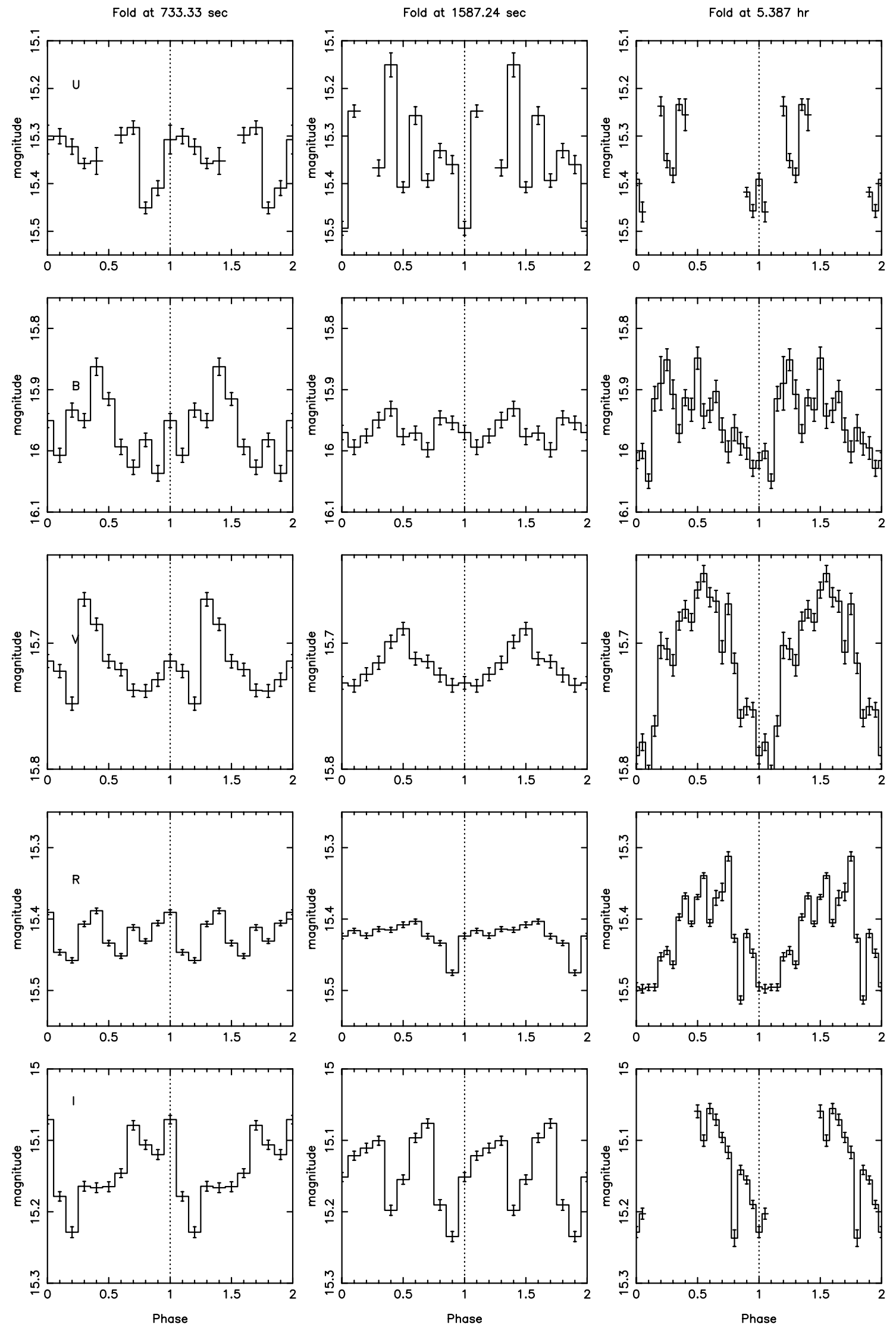

Fig. 7. The JKT $U, B, V, R$ and $I$ lightcurves of 1WGA J1958.2+3232 folded at half the spin period (left column), at the beat period (centre column) and at the orbital period (right column). Profiles are shown repeated over two cycles and phase zeros (minimum light) are according to the ephemerides given in the text. 

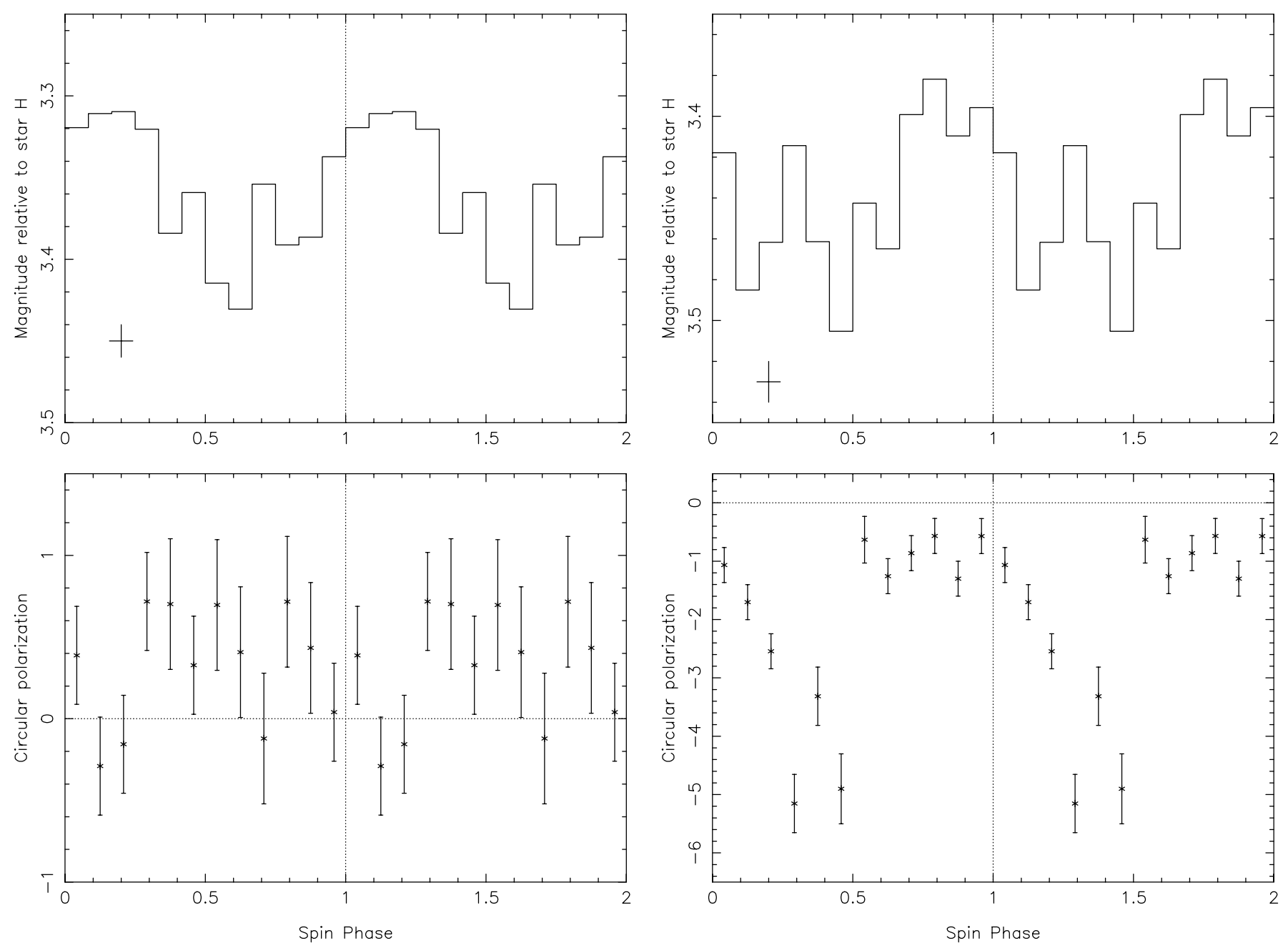

Fig. 8. The $B$-band photopolarimetry data of $1 \mathrm{WGA}$ $\mathrm{J} 1958.2+3232$ folded at $1466 \mathrm{~s}$ and repeated over two spin cycles. Panel a) shows the relative photometry with respect to star H. The typical error bar shown is estimated from the standard deviation of the magnitude of star $\mathrm{H}$ with respect to another star of constant brightness in the field. Panel b) shows the percentage circular polarization with one-sigma error bars.

since the data span only just over two spin cycles, the profiles may not be as representative as other more extensive data sets.

The phase binned $B$-band curves (Fig. 8) show that the circular polarization is about $+0.6 \%$ between spin phases 0.3 and 0.8 , contemporaneous with a 0.1 mag fading, whilst the rest of the time the polarization is close to zero. The mean circular polarization is $+0.32 \% \pm 0.10 \%$, a clear $3 \sigma$ detection. In the $R$-band, the circular polarization is negative (Fig. 9b) with a mean level of $-1.99 \% \pm 0.11 \%$. At phase 0.0 the level of polarization is around $-1 \%$ and the level increases to $-5 \%$ (near spin phase 0.4). Just after phase 0.5 the circular polarization drops quickly to the $-1 \%$ level.

We also note that the circular polarization light curves of the flux comparison star $\mathrm{H}$ are consistent with zero in both wavebands, varying typically between $\pm 0.1 \%$ in both $B$ and $R$.

Fig. 9. The $R$-band photopolarimetry data of $1 \mathrm{WGA}$ J1958.2+3232 folded at $1466 \mathrm{~s}$ and repeated over two spin cycles. Panel a) shows the relative photometry with respect to star H. The typical error bar shown is estimated from the standard deviation of the magnitude of star $\mathrm{H}$ with respect to another star of constant brightness in the field. Panel b) shows the percentage circular polarization with one-sigma error bars.

\subsection{X-ray pulsation}

Figure 10 shows the power spectrum of the $A S C A$ X-ray lightcurve. A strong signal is clearly detected at a frequency of $2 \omega$, but no other harmonics or frequencies related to either the orbital or beat frequencies of 1WGA J1958.2+332 are seen. The ASCA data in two energy bands folded at both the pulse period and twice the pulse period (i.e. the spin period of the white dwarf) are shown in Figs. 11 and 12. There is only a slight difference between the two pulses apparent in Fig. 12, as might be expected from the lack of a sub-harmonic in the power spectrum. 1WGA J1958.2+3232 appears to be a classic "double-peaked" pulse profile intermediate polar. The modulation depth is close to $100 \%$ in the soft energy band, and is less in the hard band, indicating that photoelectric absorption is a major contributor to the overall profile. There are indications of some structure to the pulse 

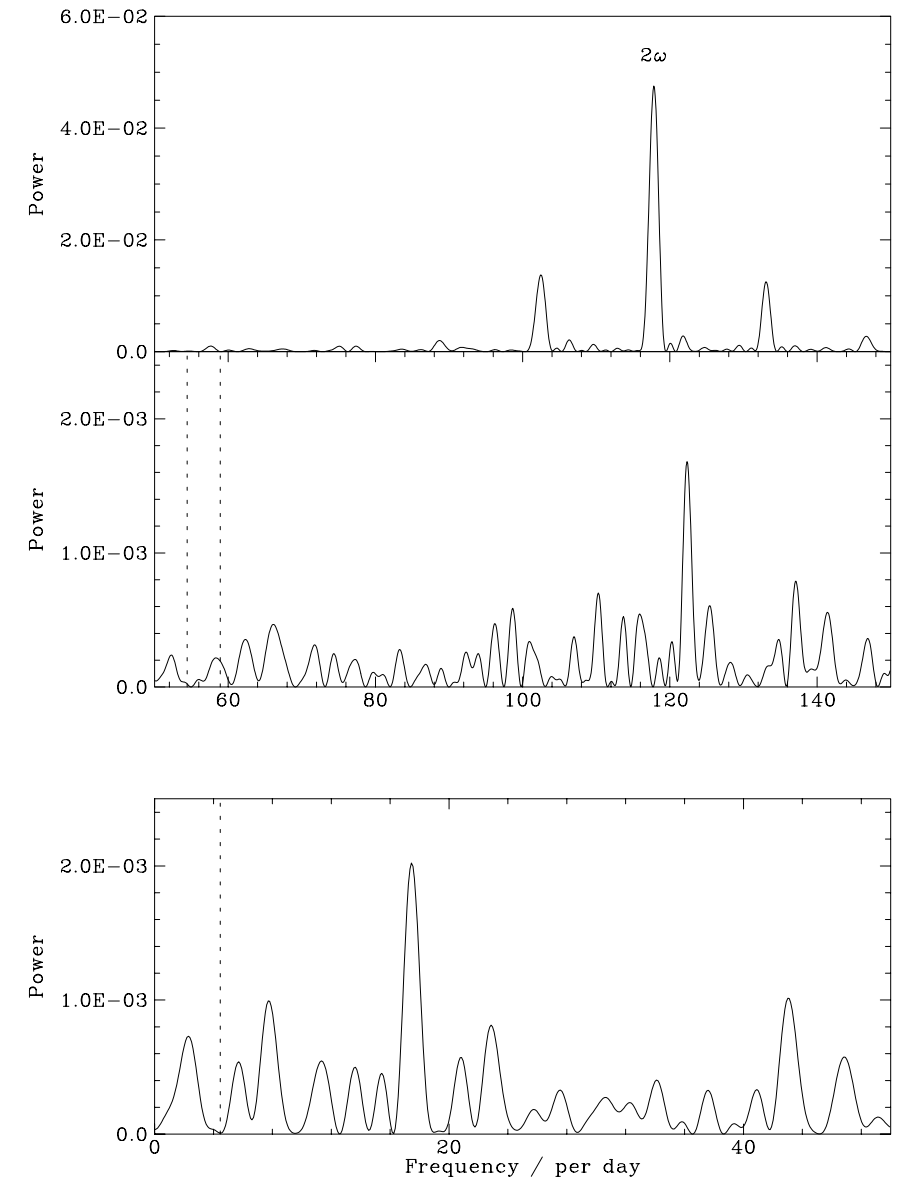

Fig. 10. The power spectrum of the total $(0.4 / 0.7-10 \mathrm{keV})$ ASCA light curve of 1WGA J1958.2+3232. The top panel shows original power spectrum in the frequency range 50$150 \mathrm{~d}^{-1}$. The dominant signal is at a frequency of $2 \omega$ with sidebands due to the spacecraft orbital frequency. The middle panel shows the same frequency range, after the dominant signal at $117.82 \mathrm{~d}^{-1}(2 \omega)$ has been removed (note the change in vertical scale). The two dashed lines show the locations of the spin and beat frequencies derived earlier. The bottom panel shows the power spectrum in the $0-50 \mathrm{~d}^{-1}$ range, after the same prewhitening. The dominant signal in this panel is found near the spacecraft orbital frequency. The dashed line here shows the location of the orbital frequency identified earlier.

profiles, beyond a simple sinusoidal modulation. For instance, the "notches" apparent near the pulse peaks are reminiscent of those seen in AO Psc and V1223 Sgr (Norton 1995). However, in this case they are only marginally significant given the error bars shown in the profiles.

\section{Discussion and summary}

Optical photometry from the JKT has allowed us to confirm the detection of an optical pulsation in 1WGA J1958.2+3232 at a period of $733.33 \mathrm{~s}$ and define a refined ephemeris. $A S C A$ observations also confirm the presence of an X-ray pulsation at the same period as seen in the optical. Our detection of a second optical pulsation period of $1587.24 \mathrm{~s}$, identified with the beat period of the

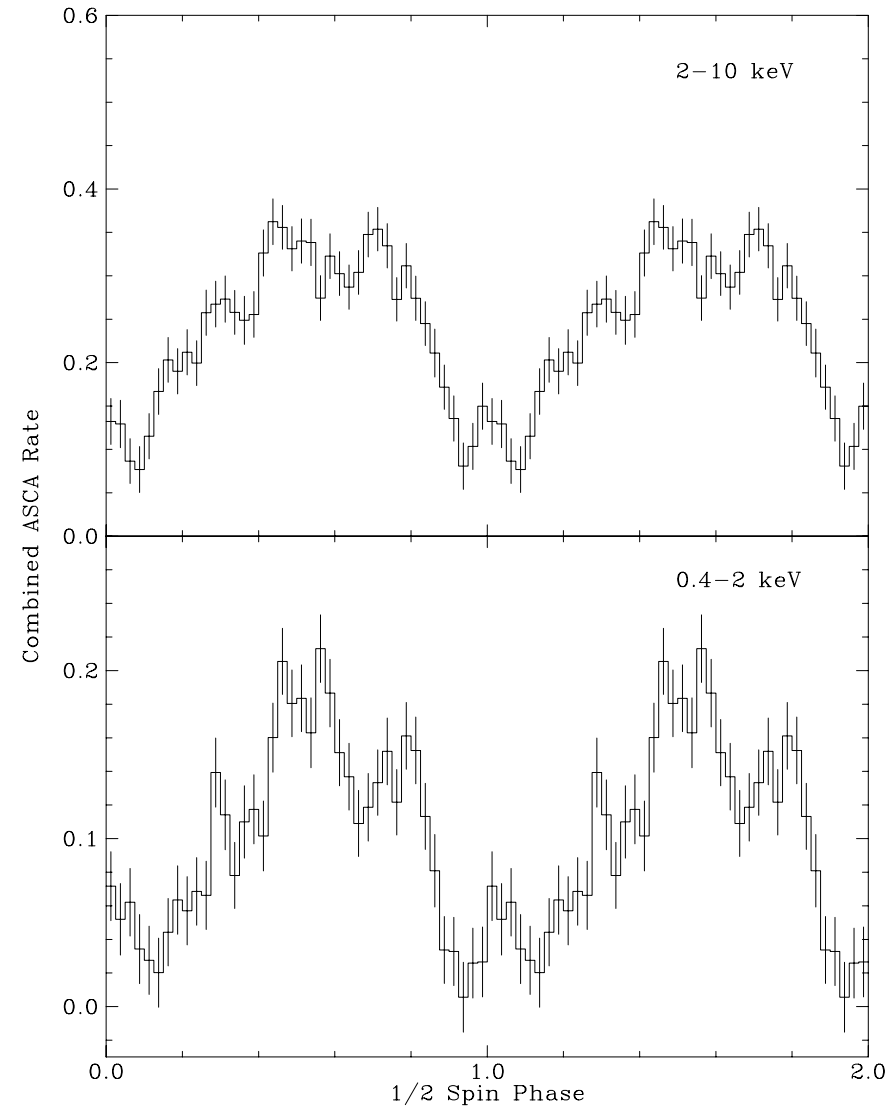

Fig. 11. The hard and soft ASCA lightcurves of $1 \mathrm{WGA}$ $\mathrm{J} 1958.2+3232$ folded at the pulse period $733.33 \mathrm{~s}$.

system, implies that the spin period of the white dwarf is twice the short pulsation period, i.e. $P_{\text {spin }}=1466.66 \mathrm{~s}$ and that the orbital period is the -1 day alias of the strongest low frequency peak in our power spectrum, i.e. $P_{\text {orb }}=5.387 \mathrm{~h}$. This is also the -1 day alias of the previously reported orbital period. We detect an orbital modulation in each of the $U, B, V, R$ and $I$ bands for the first time.

We also confirm the presence of circularly polarized emission from this source, which is thus only the fifth IP to exhibit such behaviour. The circular polarization is negative in the $R$-band and positive in the $B$-band, and in each case shows evidence for variation across the spin cycle. The level of polarization in the $R$-band, at $\sim-1 \%$ to $\sim-5 \%$, is greater than that seen by Uslenghi et al. (2000) who saw a mean level of $\sim-0.5 \%$, whilst our detection of positive circular polarization in the $B$-band is a first detection in that band.

Since the spin period of the white dwarf is twice the pulsation period observed in both optical and X-ray flux, the pulse profile is actually double peaked. Following Norton et al. (1999), a double peaked X-ray pulse profile indicates that two-pole accretion in 1WGA J1958.2+3232 conspires to produce two peaks per revolution of the white dwarf. In this model, the white dwarf has a relatively low magnetic field strength, and consequently an accretion disc which is truncated only relatively close to the 


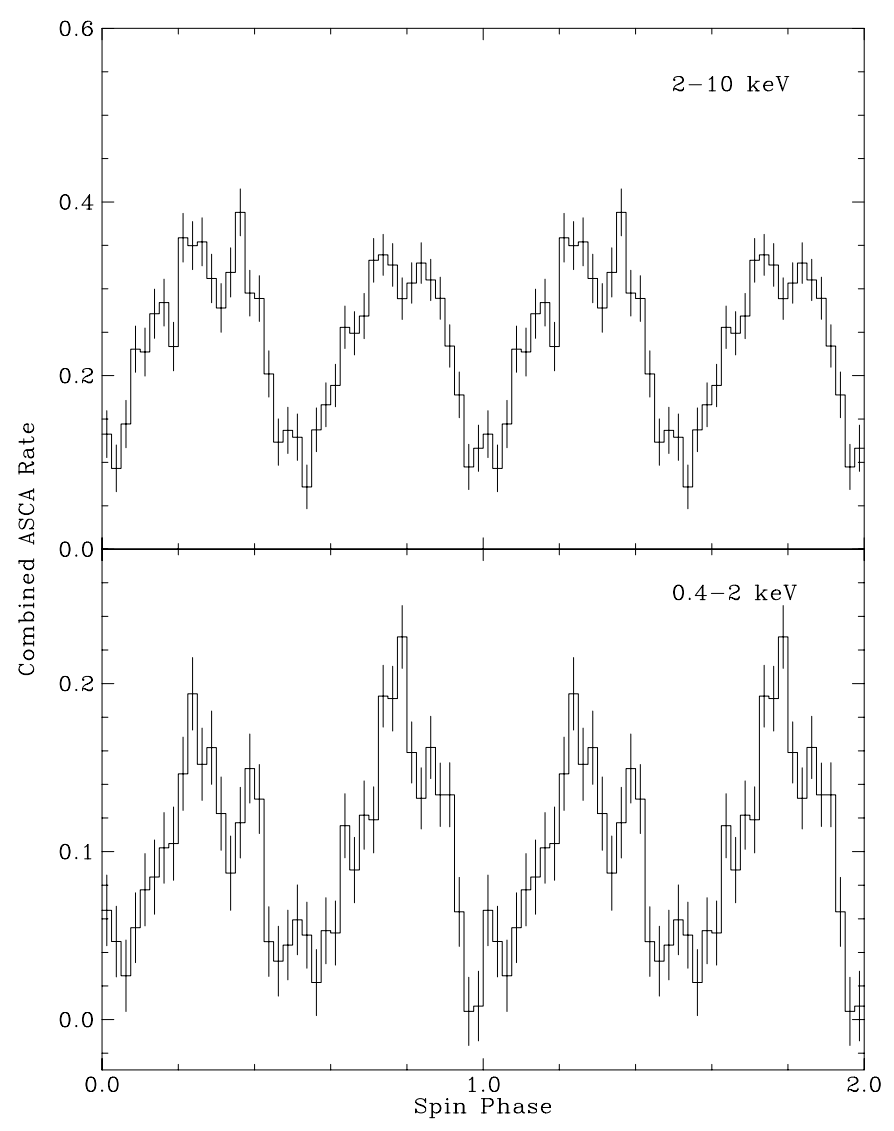

Fig. 12. The hard and soft $A S C A$ lightcurve of $1 \mathrm{WGA}$ J1958.2+3232 folded at the white dwarf spin period $1466.66 \mathrm{~s}$.

white dwarf. This leads to large footprints of the accretion curtains and an optical depth to X-rays across the accretion regions which is largest in a direction parallel to the white dwarf surface, and smallest perpendicular to the surface. (This is the reverse of the standard accretion curtain model which leads to a single peaked X-ray pulse profile.) Confirmation of this model would require pulse phase resolved optical spectroscopy in order to measure the direction of flow of the accreting material corresponding to the phases of pulse maximum and minimum. Since this model also predicts that the accretion flow is essentially via a disc, an X-ray beat modulation would not be expected to be present, in accord with all observations so far, including the $A S C A$ data presented here.

The different polarities of the circular polarization observed in the $B$ - and $R$-band data, together with light curve variations, also suggest two pole accretion. We suggest that between spin phases 0.0 and 0.5 the negative polarization accretion pole is seen, and after phase 0.5 emission from this pole reduces. Between spin phase 0.3 and 0.8 , the positive polarization accretion pole is seen in the $B$-band. The different colours of the two accretion poles may indicate that the poles have significantly different magnetic field strengths, possibly as a result of an offset dipole field structure. We note however, that the detection of circular polarization from an IP is generally taken to be an indication of a relatively strong magnetic field, in contradiction to the implication of the doublepeaked pulse profile model. There is though no evidence for a strong magnetic field in this system. The figure of $B=8 \mathrm{MG}$ stated by Uslenghi et al. (2000) is merely a value chosen for illustrative purposes and is in no way "derived" from their polarization measurements. We suggest that the magnetic field strength of 1WGA J1958.2+3232 is low enough to allow a small disc truncation radius, and consequently produce a double peaked X-ray pulse profile, but high enough that geometrical effects allow a detection of polarized emission. For instance, in a system with a more symmetrical field pattern than is implied here, the (positive) polarization from one pole may be effectively cancelled out by that (negative) from the other pole, so yielding a net polarization close to zero and hence undetectable. It may be that the only reason we detect polarization in 1WGA J1958.2+3232 is that the two poles have sufficiently different emission properties that a net polarization remains. In this interpretation, the question of whether each IP exhibits polarized emission is not only dependent upon the strength of the white dwarf magnetic field, but also on the geometry of the system and that of its magnetic field.

Acknowledgements. The data analysis reported in this paper was carried out using facilities provided by PPARC, Starlink and the Open University Research Committee. Astronomical computing at the $\mathrm{OU}$ is supported under PPARC grant PPA/G/O/2000/00037; HQ was also supported by PPARC research grant GR/L64621 during this work. SK wishes to thank the Finnish Academy of Sciences and Letters (Academia Scientiarum Fennica) for support. HL wishes to acknowledge support from grants 71355 and 44011 by the Finnish Academy. The authors thank Ian Howarth for acquiring and reducing the optical spectra reported herein. The Jakobus Kapteyn Telescope and Isaac Newton Telescope are part of the Isaac Newton Group of Telescopes on the island of La Palma. They are operated on behalf of the UK Particle Physics and Astronomy Research Council and the Netherlands Organisation for Scientific Research, in the Spanish Observatorio del Roque de los Muchachos of the Instituto de Astrofísica de Canarias. The Nordic Optical Telescope is operated on the island of La Palma jointly by Denmark, Finland, Iceland, Norway and Sweden, in the Spanish Observatorio del Roque de los Muchachos of the Instituto de Astrofísica de Canarias. The polarization data presented here have been taken using ALFOSC, which is owned by the Instituto de Astrofísica de Andalucia (IAA) and operated at the Nordic Optical Telescope under agreement between IAA and the NBIfAFG of the Astronomical Observatory of Copenhagen. This research has made use of data obtained from the High Energy Astrophysics Science Archive Research Center (HEASARC), provided by NASA's Goddard Space Flight Center. IRAF is written and supported by the IRAF programming group at the National Optical Astronomy Observatories (NOAO) in Tucson, Arizona. NOAO is operated by the Association of Universities for Research in Astronomy (AURA), Inc., under cooperative agreement with the National Science Foundation. 


\section{References}

Hellier, C. 1995, in ASP Conf. Ser. 85, Proc. Cape Workshop on Magnetic CVs. Astron. Soc. Pac., ed. D. A. H. Buckley, \& B. Warner (San Francisco), 185

Hellier, C. 1996, in Cataclysmic Variables and Related Objects, ed. A. Evans, \& J. H. Woods (Kluwer Academic Publishers, Dordrecht), 143

Israel, G. L., Angelini, L., Campana, S., et al. 1998, MNRAS, 298,502

Israel, G. L., Covino, S., Polcaro, V. F., \& Stella, L. 1999, A\&A, 345, L4

King, A. R., \& Williams, G. A. 1983, MNRAS, 205, 57P

Negueruela, I., Reig, P., \& Clark, J. S. 2000, A\&A, 354, 29

Norton, A. J. 1995, in ASP Conf. Ser. 85, Proc. Cape Workshop on Magnetic CVs. Astron. Soc. Pac., ed. D. A. H. Buckley, \& B. Warner (San Francisco), 285

Norton, A. J., Beardmore, A. P., Allan, A., \& Hellier, C. 1999, A\&A, 347, 203
Norton, A. J., Beardmore, A. P., Retter, A., \& Buckley, D. A. H. 2000, MNRAS, 312, 362

Patterson, J. 1994, PASP, 106, 209

Uslenghi, M., Bergamini, P., Catalano, S., Tommasi, L., \& Treves, A. 2000, A\&A, 359, 639

Uslenghi, M., Tommasi, L., Treves, A., Piirola, V., \& Reig, P. 2001, A\&A, in press

Stetson, P. 1992, in ed. D. M. Worrall, C. Biemesderfer, \& J. Barnes, ASP Conf. Ser. 25, Astronomical Data Analysis Software and Systems I, Astron. Soc. Pac. (San Francisco), 297

Tanaka, Y., Inoue, H., \& Holt, S. S. 1994, PASJ, 46, L37

Warner, B. 1995, Cataclysmic Variable Stars, Chap. 7 (Cambridge University Press)

Zharikov, S. V., Tovmassian, G. H., Echevarría, J., \& Cárdenas, A. A. 2001, A\&A, 366, 834 